\title{
"Internet+" Times College English Online Course Group Construction and Teaching Model Application Research
}

\author{
$\mathrm{Xu} \mathrm{Xiaoshu}$ \\ Foreign Languages College, Dalian Neusoft University of Information, Dalian, Liaoning, China \\ xuxiaoshu@neusoft.edu.cn
}

\begin{abstract}
With the rapid development of the Internet, online courses are gradually increasing on the base of the advance of the $5 \mathrm{G}$ era and the popularization of mobile learning terminals. However, in the eyes of learners, the systemic and continuity of online courses is somewhat lacking. Based on constructivism theory, a systematic and selective network courses group ought to be established with students as the center, to teach students according to their aptitude, to explore learning, and to encourage students to apply existing schemas in personalized environments to assimilate and adapt to new Information. network courses group teaching helps students to carry out active learning and enhance their ability to analyze and solve problems. The design, practice and evaluation of this courses group fully affirmed the effectiveness of the courses group, but also revealed some issues worthy of consideration and further discussion.
\end{abstract}

Keywords: Constructivism, College English, network courses group, exploratory learning

\section{“互联网+”时代大学英语网络课程组建设与教学模式 应用研究}

\author{
许晓书
}

大连东软信息学院外国语学院, 大连, 辽宁, 中国 xuxiaoshu@neusoft.edu.cn

\section{摘要}

在互联网高速发展的背景下, $5 \mathrm{G}$ 时代的来临和移动学习终端的普及, 网络课程逐渐增多, 但在学习者 看来, 网络课程的系统性和连续性却有一定的缺失。本文提出以建构主力理论为基础, 以学生为中心, 架构系统且有一定选择性的大学英语网络课程组, 因材施教、探索式学习, 鼓励学生在个性化环境中 应用已有的图式去同化和顺应新的信息。大学英语网络课程组教学有助于学生开展主动学习, 增强分 析问题和解决问题的能力。该课程组的设计、实践和评估充分肯定了大学英语网络课程组的成效, 但 也揭示了一些值得思考和进一步探讨的问题。

关键词: 建构主义，大学英语，网络课程组，探索式学习

\section{1. 前言}

随着社会的全面发展, $5 \mathrm{G}$ 时代的到来，电脑和手机 终端等信息技术设备的基本普及, 信息化时代已经悄然 来临。”互联网+”迅速发展的背景下, 实现教育信息化、 教育现代化已经成为不可逆转的发展趋势, 信息时代催 生了"互联网+"背景下的网络教学模式, 并且在高等教 育实践中获得了广泛的青崃。2020 年以前, 基于互联网 的微课、慕课等翻转课堂教学模式已经逐渐在各个高等 院校的英语课堂得以广泛的应用。

\section{2. 建构主义的理论基础}

建设系统的大学英语网络课程组其理论基础来自 近代著名的教育理 论之一，建构主义 (constructionism)。瑞士著名心理学家皮亚杰因其创 立的关于儿童认知发展理论, 是当代建构主义理论的最 早提出者。其后，科尔伯格对认知结构的性质与认知结 构的发展条件等方面的进一步研究，斯滕伯格和卡茨对 认知过程中如何发挥个体主动性的探索, 维果茨基创立 
的强调认知过程中学习者所处的社会历史文化背景作 用的 “文化历史发展理论”, 布鲁纳的发现学习理论 等研究成果和理论观点为当代建构主义的形成奠定了 基础。

建构主义学习理论认为: 学习是一个积极主动的建 构过程。学习者不是被动地接受外在信息, 而是根据先 前认知结构主动地和有选择性地知觉外在信息, 建构当 前事物的意义。最理想的学习者是会自我调节的学习者, 这种学习者能够掌握有效的学习策略, 并且根据自身经 验和社会感知等因素能做出及时反映, 适时地运用掌握 的学习策略。因此, 在网络课程组的设计中, 教师通过 鼓励学生自身去发现概念和原理, 从而实现自我学习。 探索式学习可以引起学生的兴趣, 唤起好奇心, 鼓励学 生自己努力, 直至发现答案。同时培养学生独立分析问 题、解决问题和总结问题等一系列批判性思维的能力。

\section{3. 大学英语网络课程的现状}

目前看来, 我国各大高校所开展的大学英语网络课 程主要分为以下两种: 第一类为各个高校根据自身的培 养目标, 结合学生的自身特点, 由大学英语教师组织设 计的大学英语网络课程, 此类课程比较符合各高校学生 的专业特点和水平, 但受到教师水平、技术水平等影响, 具有一定的随意性和偶然性, 相对来说影响范围较小, 授课范围和授课深度有限。

另一类大学英语网络课程则是由高校教学管理部 门支持和管理下统一设计、统一制作和管理的。此类课 程有国家级、省级和校级的三级精品课程体系, 网易云 课堂, 中国大学 mooc, 学堂在线等平台均提供了此类网 络课程。这种大学英语课程一般是由学科教师团队开发, 有相关的政策和技术支持, 面向广大学子, 其实施的范 围较广, 有一定的影响力, 但是因为其普适性, 所以相 对来说缺乏一定的针对性, 和高校中具体的培养目标、 教学目标等契合度不高。

长期以来, 大学英语课程设置以基础课程为主, 必 修课程比重大，学生自由选择课程的空间狭小，而且 基础性课程并不能够全面反映高等院校学子其英语基 础及发展方向的语言需求和发展状况。且在目前的网络 课程教学研究中, 其重点大多数在于教学内容设计, 网 课技术的应用与开发、学生学习的支持辅助等方面, 但 是对于如何规划并管理系统的网络课程组群及其相关 资源, 如何进行有效的课程评价, 此部分目前研究较少, 有待于进一步的研究和深入探讨。

在高校的实际教学过程中, 存在着各高校的大学英 语网络课程常常比较独立, 且资源更新速度稍慢, 交互 程度较低等问题。因此如何进一步整合大学英语课程组 与常规教学资源, 提高师生交互程度, 逐渐改变 “教师 为中心” 的教学观念, 以及培养学生对于网络环境的适 应程度, 改善学生的学习习惯等方面, 我们可以逐渐研 究探讨, 逐一加以完善和提升。目前多数高等院校英语 课程仅靠一门网络课程教学, 效果欠佳。如能形成多门 课程相互支撑、合理组合的网络课程组, 则更有效地实
现大学英语的教学目标，完善并丰富教学内容，整合教 学资源, 体现大学英语特色, 有效提升课程评价和学习 效果, 并有利于促进高校学生英语水平。

\section{4. 大学英语网络课程组的建设}

大学英语网络课程组即是根据学生的培养目标和 培养方案, 打破原有课程内容的归属性, 将英语教学中 内容相关性较强, 关联逻辑性较强的同一范畴的网络课 程作为一个整体系统的课程组, 在教学目标设置、内容 设计与选取、资源建设与完善、教学评价等方面实现课 程组的整体评价, 从技能培养目标层次把握课程内容的 分配、实施、保障和技能的实现。可以采用多科联考, 考核学生的整体英语水平与应用能力。

以大连某高校建设的大学英语课程组为例, 基于前 期的问卷调查和随机访谈结果, 系统调研国家级、省级 及若干院校的大学英语网络课堂教学内容和方式, 通过 对材料进行分析总结, 进一步整合大学英语网络课程体 系。该课程组在层级上分为基础和综合：基础课程为: 语法、词汇课程; 技能课程为: 阅读、翻译、写作、听 说课程; 核心课程为综合英语; 高阶课程为英语国家文 化，跨文化交际。资源建设上有四个维度：课件库、试 题库、案例库和项目库。通过课程资源管理系统和远程 教学支持系统实现学习者的课程使用。各课程教学内容 具有不可重复性, 同时知识点之间存在相对独立和离散 性, 知识点之间关系亲和, 内容可集群。课程组织内部 的项目实践环节或技能培养环节是系统连贯的, 逐层递 进的, 根据当代社会的实际现状和毕业后职业实际应用 所设计。

大学英语网络课程组主要通过汶览器作为学习的 终端, 组织形式主要以树状目录导航, 超级链接连接各 个学习模块, 课程资源的形式多样, 有文字说明、视频 音频、图像图形等等。基本学习素材有课程导学, 单元 导学, 学习重点与难点分析, 主题相关词汇, 教学视频, 阅读材料, 测试习题, 讨论区, 拓展资源等等, 构建了 系统有效又有针对于专业特色的优质大学英语课程组, 为学生提供更为宽厚的有针对性的大学英语课程体系, 为进一步推动大学英语网络课程的开发与应用提供了 广阔的思路。此大学英语网络课程组的特色有以下几点:

\section{1. 统一的网络课程建设标准}

教育信息化技术委员会发布的 “教育信息化技术 标准”（或称网络课程评价规范）是大学英语网络课程 组建设所依据的国家标准。大学英语网络课程组建设在 设计和开发过程中时刻参考标准, 集思广益, 注重整体 概念和理解, 保证了网络课程组的建设质量。该标准是 针对高等教育、基础教育以及职业教育中建设的网络课 程所提出的, 它是对网络课程质量进行评价的指标体系 和评价指南。

根据该标准, 网络课程要提供充分的资源扩展, 资 
源扩展是网络课程中与课程内容相关的、有学习价值的 外部资源链接和参考资料。资源扩展包括 “在线资源和 离线资源，在线资源主要包括但不限于相关网络课程、 专业学科网站、相关网上图书与杂志、著名研究者或研 究机构的主页、相关的政府网站等”。

\section{2. 多层级的教学目标}

根据校本学生专业的培养目标和大学英语教学指 南, 结合最新推出的 “外语等级量表”, 大学英语网络课 程组的教学目标与学生的专业培养目标一致, 根据学生 的专业特点和英语水平, 量身定制, 以面向工科专业学 生的大学英语网络课程组为例, 根据培养目标及学生的 职业期许, 该系列的大学英语课程更注重学生的阅读和 写作能力, 听说次之, 根据学生的高考分数、入学考试 分数和每学期的英语能力水平测试, 学生分为 $\mathrm{ABC}$ 三个 层次, 而每个层次的教学目标和对应的教学内容又是有 所侧重, 有所不同的。

\section{3. 丰富实际的教学设计}

以前的很多大学英语网络课程仅仅是利用多媒体 技术将教学内容呈现给学生, 而大学英语网络课程组的 建设将教学设计和内容并重, 重视学习者的自主学习能 力的培养, 重视真实教学情境的创设, 通过教学输出的 目标的设定让学生以小组合作的形式去解决一系列真 实有效的问题。利用小组合作, 角色扮演, 探索式学习 等一系列教学方法将目前形式单一的网络课程丰富立 体起来。

当今的社会是学习型社会, 谁能不断进取, 拥有卓 越的学习能力和自制力谁就能拥有良好的职业素养和 综合素养。因此大学英语网络课程组更注重学生自主学 习能力的培养。该网络课程组结合当下的职业需求根据 学习的专业设置了系列的学习输出项目, 如学校概况 PPT 制作, 问卷调研等, 让学生有的放矢, 学以致用, 在项目驱动力的引导下, 自主学习知识, 搜集信息, 实 践运用知识, 完成学习的闭环。

\section{4. 持续更新的教学资源}

大学英语网络课程组的教学资源每学期全面更新 完善, 每学期教学案例库和项目库增加 5-10\%, 试题库每 个题型更新或增加 $5 \%$, 不断更新且与时俱进的课程资源 源源不断地吸引着学习者不断前行。为了便于学习者利 用碎片化时间学习, 所制作的学习视频一般在 5-10 分 钟长度, 学生下载和在线观看都流畅清晰。同时, 基于 学生的专业不同, 水平层次不同, 为 $\mathrm{C}$ 层次的同学提供 了系列的语法课程, 为 A、B 层次的同学提供了文化拓 展学习内容, 并给予了有针对性的自学指导, 有课程导 学和单元导学两个层级。

且因为学习者的学习时间具有不确定性, 教师无
法监控其学习的固定时间也不能保证固定时间长度，因 此在资源建设中, 注重学习的碎片化, 因此教学视频的 长度较短, 阅读文章篇幅处理也比较灵活。关注学生个 性差异，提供多种可供选择的学习资源。当然在注重多 样性的同时, 并非给予学习者的资源数量和类型越多越 好, 这样容易造成学习者认知超载, 或分散学习者的注 意力。

\section{5. 学习者友好型的导航与环境}

为了有效地引导学生完成网络课程学习, 课程组按 照教学进程表设计了较为清晰, 简单易懂的导航系统, 可以根据学习时间和学习单元比较快速地定位到学生 要学习的相关知识, 菜单层级分明, 链接层次明确, 学 习者登录后基本点击 2-3 次就可以打开相关的网络课程, 清晰流畅地进行网络学习和网络互动。大学英语网络组 的系列课程在 Win7、Win10、Mac 等系统下，一般的浏 览器上均可播放, IE、谷歌、火狐、360 等, 一般不用 设置兼容性等问题, 使学生在疫情期间在家及时有效地 完成大学英语的课程学习。

\section{6. 多维度的教学评价和反馈机制}

较之以前单一的网络课程评价方式, 所建设的大学 英语网络课程组采用了较为全面系统多维度的评价体 系, 采取定量评价与定性评价相结合的方法来提高评价 结果的可靠性与可比性。每个课程小节都附带了一个学 生自测, 学生完成相关作业后首先系统评价, 之后学习 者互评, 最后教师对于学生提交的作业进行评价、反馈, 经过反馈后的作业学生再次修改完善。课程学习完毕后 除整体的针对性内容测评外还有一个第三方网站进行 英语水平测试, 全面衡量学生的内容学习和水平提升。

通过这样系统的评价机制, 学生能及时了解自己的 学习效果和横向比较的情况。课程重视前期建设, 更重 视其后期应用与反馈评价。

\section{5. 结论}

大学英语网络课程组建设为特殊时期的高校学子 进行远程学习提供了全面的支持和服务, 辅助提升大学 英语课程的教学质量。但同时在课程建设、资源设计与 开发, 后续评价反馈等环节一定还存在些许瑕疵, 例如 目前该课程组学习系统还缺少搜索定位功能, 不能快速 查找某个具体的知识点或者课程资源, 还需要进一步完 善。因此, 系统而完善的大学英语网络课程组的开发和 建设需要各个方面的智者的参与, 有来自教学一线的大 学英语教师, 有研究教学教法的学科专家, 有卓越的计 算机网络技术人员等, 经过反复研究实践和共同合作, 才可以创造出精品的网络课程组资源, 充分满足学习者 的个体需要, 促进终身教育体系和学习型社会的发展和 完善。 


\section{REFERENCES}

[1] Paris, S.G., Paris, A.H. (2001) Classroom Applications of Research on Self-regulated Learning. J. Educational Psychologist, 180:57-62.

[2] Stoller, F. L. (1997) Project work: A means to promote language and content. J. English Teaching Forum, 195:31-37.

[3] Thomas, R. W., McGregor, S. K. (2005) Online project-based learning: How collaborative strategies and problem solving processes impact performance. J. Journal of Interactive Learning Research, 233: 83-107.

[4] Xu Xiaoshu. (2019) An Empirical Study of the Instrument for the Project-based College English Classroom Environment Construction and Evaluation. In: Proceedings of the 2nd Symposium on Health and Education. Shanghai. pp. 478-482 